\title{
Os benefícios da atuação do fisioterapeuta em doenças perinatais
}

A paralisia cerebral (PC), atualmente definida por encefalopatia crônica infantil não progressiva, é caracterizada por uma agressão ao sistema nervoso central (SNC), e é descrita por distúrbio do movimento, tônus e postura, tendo como principal alteração o comprometimento motor e mudanças na mecânica corporal, podendo apresentar alterações sensitivas, visuais, intelectuais e auditivas. O objetivo dessa revisão bibliográfica foi verificar os benefícios da atuação do fisioterapeuta em doenças perinatais, demonstrando a contribuição da fisioterapia na recuperação de pacientes em doenças perinatais, como a paralisia cerebral. Através dos estudos, compreendeu-se melhor as causas, prevenção e tratamento da paralisia cerebral, como também observou-se que o paciente com PC pode sofrer influencias de fatores de risco que interferem em seu desenvolvimento motor, prejudicando o comprometimento de suas habilidades funcionais de vida diária e função social.

Palavras-chave: Doenças perinatais; Fisioterapia em paralisia cerebral; Paralisia cerebral; Estimulação precoce.

\section{The benefits of the physiotherapist's performance in perinatal diseases}

Cerebral palsy (CP), currently defined as chronic non-progressive childhood encephalopathy, is characterized by an aggression to the central nervous system (CNS), and is described by movement disorder, tone and posture, with the main alteration being motor impairment and changes in the body mechanics, being able to present sensitive, visual, intellectual and auditory alterations. The objective of this literature review was to verify the benefits of the physiotherapist's performance in perinatal diseases, demonstrating the contribution of physiotherapy in the recovery of patients in perinatal diseases, such as cerebral palsy. Through the studies, it was better understood the causes, prevention and treatment of cerebral palsy, but it was also observed that the patient with CP can be influenced by risk factors that interfere in their motor development, impairing the impairment of their functional abilities of daily life and social function.

Keywords: Perinatal diseases; Physiotherapy in cerebral palsy; Cerebral palsy; Early stimulation.

\section{Topic: Fisioterapia}

Reviewed anonymously in the process of blind peer.
Received: 20/08/2019

Approved: 27/08/2019
Gelmária Nunes Pereira

Faculdade Guaraí, Brasil

http://lattes.cnpq.br/8580272532769954

gelmaria@gmail.com

Claudiane Sousa Costa

Faculdade Jangada, Brasil

http://lattes.cnpq.br/2203896732398916

clausol.costa@hotmail.com

Karla Camila Correia da Silva

Faculdade Guaraí, Brasil

http://lattes.cnpq.br/1981447087125364

karlacamilac@yahoo.com.br

\section{Referencing this:}

PEREIRA, G. N.; COSTA, C. S.; SILVA, K. C. C. S.. Os benefícios da atuação do fisioterapeuta em doenças perinatais. Scire Salutis, v.8 n.2, p.115-122, 2018. DOI: http://doi.org/10.6008/CBPC22369600.2018.002.0012

DOI: 10.6008/CBPC2236-9600.2018.002.0012 


\section{INTRODUÇÃO}

Em 1843, o médico inglês William John Little observou vários casos de crianças com rigidez muscular que nasceram prematuras, ou que tiveram complicações no parto e que apresentavam maior acometimento nos membros inferiores como também, dificuldades neuropsicomotoras, sendo a principal causa de dano cerebral, a anóxia. A essa condição, foi denominada doença de Little, mais conhecida como Diplegia Espástica ou PC (MADEIRA et al., 2009).

Sigmund Freud, em 1897, analisou os estudos de Little, e passou a usar o termo PC, questionando se as anormalidades quanto ao processo de nascimento seriam por fatores etiológicos que ocorriam apenas durante o parto ou por causas pré-natais. No entanto, a expressão PC só passou a ser consagrada e generalizada em 1946 por Phelps (SANTOS, 2014).

De acordo com Ferreira (2016), PC atualmente definida por encefalopatia crônica infantil não progressiva, é caracterizada por uma agressão ao SNC é descrita por distúrbio do movimento, tônus e postura, tendo como principal alteração o comprometimento motor e alterações na mecânica corporal, podendo apresentar alterações sensitivas, visuais, intelectuais e auditivas. Monteiro et al. (2011) cita que a classificação da paralisia cerebral se dá de acordo com o tipo e localização da alteração motora, sendo elas: espástica, discinética, atáxica, hipotônica e mista. Na qual a forma Espástica é a mais comum e é dividida em diparéticas, hemiparéticas e quadriparéticas.

A paralisia cerebral sucede nos períodos pré, peri e pós-natal tendo como fatores etiológicos na fase pré-natal, segundo Medeiros et al. (2015), os causados por doenças como rubéola, doença von wildebrand, prematuridade, sífilis, toxoplasmose, citomegalovírus, HIV. Zanini et al. (2009) enfatiza outros fatores como doença tireoidiana materna, traumatismos abominais severos ou quedas sentada da gestante, drogas ilícitas, baixo peso, pré-eclâmpsia grave, hemorragia anteparto moderada a grave, anormalidades morfológicas da placenta, medicações especificas e doença viral, apesar destes fatores.

Embiruçu et al. (2015) cita que as classes sociais mais altas possuem maiores condições de assistência médica nos períodos pré e perinatais, diminuindo a mortalidade. No entanto, quanto aos casos de PC em comunidades de baixa renda, os cuidados básicos das gestantes no período pré-natal são menores, podendo elevar a alta taxa de mortalidade dos RNs e aumentando os casos de PC, portanto, a partir de um pré-natal bem feito e uma abordagem perinatal eficaz, alguns fatores de risco da PC poderão ser reduzidos, evitando o baixo peso ao nascer e a prematuridade sendo uma das principais causas.

No período perinatal, ou seja, durante o nascimento da criança, os fatores contribuintes para a paralisia cerebral são parto instrumentado, período expulsivo prolongado, prolapso do cordão umbilical, deslocamento prematuro da placenta, ruptura uterina, parada cardiorrespiratória da gestante, cesariana de emergência, apresentação occipto posterior, hipertermia intraparto.

E, por último, os fatores pós-natais que são má-formação dos vasos sanguíneos durante o amadurecimento do cérebro do bebê, concentração sérica elevada de bilirrubina que pode levar a uma lesão cerebral, sepse neonatal, meningite ou encefalite, má formação congênita, baixo peso ao nascer, problemas 
respiratórios primários, traumatismo craniano, tumores, afogamento, exposição ao chumbo, parada cardíaca durante cirurgias, trombose por anemia falciforme (MEDEIROS et al., 2015).

A etiologia da paralisia cerebral, segundo Silva et al. (2009), é multifatorial com origem anatomofuncional, fisiopatológica, genética, ambiental e infecciosa. A incidência e prevalência da PC, segundo Guimarães et al. (2014), consta de 1,5 a 5,9 por cada 1.000 nascidos vivos nos países desenvolvidos; porém, não há pesquisa específica que determina o número exato da incidência de casos de PC no Brasil. Em recém-nascidos a termo, a prevalência de PC é de 1,29 por 1.000 nascidos vivos nas classes sociais mais altas e 2,42 por 1.000 nascidos vivos de classes sociais mais baixas, observando que, entre os mais pobres, o peso ao nascimento é baixo ou muito baixo para a idade gestacional (EMBIRUÇU et al., 2015).

De acordo com Ávila et al. (2015), o tratamento fisioterapêutico é paliativo, devendo começar o mais precocemente para que possa ser trabalhado a plasticidade neuromotora, melhorando assim, a capacidade funcional através da estimulação de movimentos. Feitosa et al. (2016) enfatiza que a intervenção fisioterapêutica deve ter como objetivo a normalização do tônus muscular, inibir reflexos anormais, facilitar movimentos normais, promover maior grau de independência, evitar contraturas, prevenir alterações posturais e deformidades ósseas nas extremidades.

A toxina botulínica é uma neurotoxina produzida pela bactéria anaeróbica Clostridium botulinum, que age de forma eficaz prevenindo as deformidades secundárias e diminuindo a espasticidade. Também como tratamento pode-se utilizar as cirurgias ortopédicas a fim de preservar a função e aliviar a dor (TELES et al., 2011; FEITOSA et al., 2016).

Um dos métodos mais antigo, segundo Gomes et al. (2013), porém mais utilizado no tratamento fisioterapêutico, é o Método Bobath, que se baseia na inibição dos reflexos primitivos e padrões patológicos de movimentos, pois proporciona uma experiência sensorial de movimento normal através da diminuição da hipertonia, facilitação de padrões posturais e motores normais como também da funcionalidade. 0 método Kabat, de acordo com Ávila et al. (2015), também é bastante usado, pois se utiliza de estímulos proprioceptivos facilitadores de respostas reflexas e chegando à motricidade voluntária. Esta revisão de literatura teve como objetivo, verificar os benefícios da atuação do fisioterapeuta em doenças perinatais, demonstrando à contribuição da fisioterapia na recuperação de pacientes em doenças perinatais, como a paralisia cerebral.

\section{MATERIAIS E MÉTODOS}

A presente pesquisa se trata de uma revisão de literatura que busca demonstrar a contribuição da fisioterapia em pacientes com sequelas de doenças perinatais. A coleta de dados foi realizada no período de dezembro de 2017 a fevereiro de 2018, selecionando-se livros e artigos em conformidade com o assunto proposto. Para isso, realizou-se um levantamento bibliográfico nas bases de dados eletrônicas: Biblioteca Virtual em Saúde (BVS), Physiotherapy Evidence Database (PEDro), e Scientific Eletronic Library Online (SCIELO). 
Foram utilizados os descritores: 'doenças perinatais'; 'fisioterapia em paralisia cerebral'; 'paralisia cerebral'; e 'estimulação precoce'. A pesquisa incluiu apenas estudos publicados no período de 2008 a 2016 e limitou-se aos idiomas português, inglês e espanhol, sendo excluídos todos os artigos incompletos, resumos, bem como aqueles que não possuem fundamentação cientifica e/ou que não estiveram relacionados ao tema proposto. Com o auxílio dos descritores encontrou-se 37 artigos. Após a aplicação dos filtros: data de publicação (2008-2016), idiomas (inglês, português e espanhol) e textos completos, e após análise criteriosa dos achados, selecionou-se uma quantidade de 21 periódicos para a confecção do presente trabalho.

\section{RESULTADOS E DISCUSSÃO}

\begin{tabular}{|c|c|c|c|}
\hline Autor & Ano & Objetivo & Conclusão \\
\hline $\begin{array}{l}\text { MADEIRA, EAA; } \\
\text { CARVALHO, SG de. }\end{array}$ & 2009 & $\begin{array}{l}\text { Elaborar e analisar um levantamento } \\
\text { bibliográfico relacionado à Paralisia } \\
\text { Cerebral e possíveis fatores de risco ao } \\
\text { desenvolvimento motor. }\end{array}$ & $\begin{array}{l}\text { Pode-se compreender que a criança com PC, além da própria } \\
\text { lesão cerebral, é capaz de sofrer influências de fatores de risco } \\
\text { que interferem em seu desenvolvimento motor, levando a um } \\
\text { comprometimento em suas habilidades funcionais de vida } \\
\text { diária e função social. }\end{array}$ \\
\hline SANTOS, AF dos. & 2014 & $\begin{array}{l}\text { Fazer um resumo da literatura sobre a } \\
\text { paralisia cerebral. }\end{array}$ & $\begin{array}{l}\text { Conclui-se que a PC está diretamente relacionada a uma } \\
\text { assistência pré-natal adequada capaz de diagnosticar, e fazer } \\
\text { a intervenção necessária quando for o caso. Um pré-natal } \\
\text { contínuo e regular, com aconselhamento e nutrição materna } \\
\text { adequados, evitando o uso de teratógenos, inibe, de forma } \\
\text { considerável, a incidência de paralisia cerebral. }\end{array}$ \\
\hline FERREIRA, MTS. & 2016 & $\begin{array}{l}\text { Identificar a incidência da faixa etária, do } \\
\text { gênero, tipos e modos de PC através } \\
\text { de análises de prontuários de crianças } \\
\text { atendidas na Clínica Escola de Fisioterapia } \\
\text { do Centro Universitário Católica de } \\
\text { Quixadá no período de } 2011 \text { a } 2015 . \\
\end{array}$ & $\begin{array}{l}\text { Conclui-se que o tratamento fisioterapêutico realizado } \\
\text { precocemente a partir das observações clínicas e com base } \\
\text { nas alterações neuropsicomotoras apresentadas pela criança } \\
\text { mesmo sem diagnóstico conclusivo da patologia, o } \\
\text { prognóstico dessas crianças terá um resultado mais positivo e } \\
\text { eficaz no que diz respeito a sua evolução clínica. }\end{array}$ \\
\hline $\begin{array}{l}\text { MONTEIRO, CB de M; } \\
\text { SILVA, TD; ABREU, LC; } \\
\text { MASSA, M; LEÃO, EKEA. }\end{array}$ & 2011 & $\begin{array}{l}\text { Esclarecer algumas questões que } \\
\text { envolvem o uso da realidade virtual, assim } \\
\text { como propor áreas de pesquisa e } \\
\text { intervenções a serem desenvolvidas e } \\
\text { utilizadas com diferentes deficientes por } \\
\text { meio da utilização da realidade virtual na } \\
\text { paralisia cerebral. }\end{array}$ & $\begin{array}{l}\text { Na perspectiva de incentivar o desenvolvimento de } \\
\text { conhecimentos sobre a utilização de realidade virtual na } \\
\text { intervenção } \\
\text { de diferentes deficientes, espera-se que o presente livro } \\
\text { represente uma contribuição para os estudos acerca das } \\
\text { temáticas discutidas, considerando e respeitando que se trata } \\
\text { de um campo vasto para investigações, debates e propostas } \\
\text { de intervenção prática. }\end{array}$ \\
\hline $\begin{array}{l}\text { MEDEIROS, PAB de; } \\
\text { GRIBOSK, RA. }\end{array}$ & 2015 & $\begin{array}{l}\text { Verificar como a assistência pré-natal e o } \\
\text { parto podem influenciar/evitar a paralisia } \\
\text { cerebral }\end{array}$ & $\begin{array}{l}\text { Conclui-se que existem diferentes modelos de assistência, } \\
\text { assim como assistências consideradas inadequadas ou } \\
\text { incompletas. Porém foi possível obter dados que possibilitem } \\
\text { um atendimento individualizado, humanizado desde o } \\
\text { pré-natal com qualidade no sentido de prevenir agravos de } \\
\text { saúde no binômio mãe-bebê. }\end{array}$ \\
\hline $\begin{array}{l}\text { ZANINI, G; CEMIN, NFC; } \\
\text { PERALLES, SN. }\end{array}$ & 2009 & $\begin{array}{l}\text { Ampliar o conhecimento sobre as causas } \\
\text { para o desenvolvimento da PC, realizando } \\
\text { uma revisão da literatura, visando estudar } \\
\text { prevalência das causas nos períodos pré- } \\
\text { natal, perinatal e pós-natal. }\end{array}$ & $\begin{array}{l}\text { A pesquisa conclui que crianças com acometimento no } \\
\text { período pré-natal têm maior prevalência de desenvolver PC. }\end{array}$ \\
\hline $\begin{array}{l}\text { EMBIRUÇU, EK; } \\
\text { MONTEIRO, CB de M; } \\
\text { SILVA, TD da; REIS, } \\
\text { AOA; VALENTI, VE; } \\
\text { OLIVEIRA, AG de; } \\
\text { ABREU, LC de. }\end{array}$ & 2015 & $\begin{array}{l}\text { Discutir todas as concepções teóricas } \\
\text { ligadas a PC através do enfoque de } \\
\text { profissionais que atuam com pacientes } \\
\text { com esta patologia. }\end{array}$ & $\begin{array}{l}\text { É preciso pensar que a pessoa com dificuldades motoras } \\
\text { necessita que seu corpo tenha experiências, dentro do } \\
\text { possível, comuns, mesmo que com o motor externo. }\end{array}$ \\
\hline $\begin{array}{l}\text { SILVA, R; CAON, G; } \\
\text { RIBEIRO, J; VARGAS, CR. }\end{array}$ & 2009 & $\begin{array}{l}\text { Identificar os fatores etiológicos e avaliar } \\
\text { a função motora grossa de alunos com } \\
\text { paralisia cerebral matriculados na rede } \\
\text { pública municipal de ensino de } \\
\text { Florianópolis - Santa Catarina. }\end{array}$ & $\begin{array}{l}\text { Os menores escores na função motora grossa ocorreram nos } \\
\text { casos de quadriplegia e coreoatetose, e que as meninas } \\
\text { apresentaram escore total em valor médio maior que os } \\
\text { meninos, no entanto, esta diferença não foi estatisticamente } \\
\text { significante. }\end{array}$ \\
\hline $\begin{array}{l}\text { GUIMARÃES, CL; } \\
\text { PIZZOLATTO, TC de O; } \\
\text { COELHO, ACS; FREITAS, } \\
\text { STT de. }\end{array}$ & 2014 & $\begin{array}{l}\text { Caracterizar o perfil clínico das crianças } \\
\text { atendidas na clínica escola de fisioterapia } \\
\text { da UNIP, para uma melhor abordagem } \\
\text { terapêutica facilitando futuros } \\
\text { diagnósticos. }\end{array}$ & $\begin{array}{l}\text { Mostra o perfil clínico, epidemiológico e funcional de crianças } \\
\text { portadoras de Paralisia Cerebral assistidas pela clínica escola } \\
\text { de fisioterapia da Universidade Paulista UNIP - Campus São } \\
\text { José dos Campos, e fornecem subsídios que fundamentam as } \\
\text { estratégias de avaliação e intervenção fisioterapêutica para } \\
\text { otimização do tratamento. }\end{array}$ \\
\hline
\end{tabular}




\begin{tabular}{|c|c|c|c|}
\hline $\begin{array}{l}\text { ÁVILA, JE; RODRIGUES, } \\
\text { NS; SANTOS, RM; } \\
\text { OLIVEIRA, AKA; } \\
\text { SANTOS, ANL; SAVIAN, } \\
\text { NU. }\end{array}$ & 2015 & $\begin{array}{l}\text { Estudar os tipos de paralisia cerebral, } \\
\text { características e tratamento } \\
\text { fisioterapêutico. }\end{array}$ & $\begin{array}{l}\text { O tratamento fisioterapêutico na paralisia cerebral é de } \\
\text { grande valia para melhora do quadro do paciente. Podendo } \\
\text { também associar a outras técnicas em favor a reabilitação do } \\
\text { paciente. }\end{array}$ \\
\hline $\begin{array}{l}\text { FEITOZA, FB; PEREZ, } \\
\text { FSB. }\end{array}$ & 2016 & $\begin{array}{l}\text { Verificar os efeitos da intervenção } \\
\text { fisioterapêutica na reabilitação de } \\
\text { pacientes com paralisia cerebral } \\
\text { discinética distônica. }\end{array}$ & $\begin{array}{l}\text { A fisioterapia na Paralisia Cerebral Discinética Distônica, } \\
\text { proporciona resultados benéficos na melhora da qualidade de } \\
\text { vida geral da criança, impendido a formação de deformidades } \\
\text { devida a falta de mobilização articular, posturas prolongadas, } \\
\text { e encurtamento muscular. Estabelece metas alcançáveis } \\
\text { através de uma avaliação detalhada e individualizada, que } \\
\text { indiquem quais intervenções terão excelência no tratamento } \\
\text { de cada paciente. }\end{array}$ \\
\hline $\begin{array}{l}\text { TELES, MS; MELLO, } \\
\text { EMCL. }\end{array}$ & 2011 & $\begin{array}{l}\text { Realizar uma revisão bibliográfica por } \\
\text { meio da seleção e análise criteriosa de } \\
\text { artigos científicos que relatem os efeitos } \\
\text { da aplicação da toxina botulínica tipo A } \\
\text { associada à fisioterapia em crianças com } \\
\text { paralisia cerebral espástica. }\end{array}$ & $\begin{array}{l}\text { A TBA associada à fisioterapia para o controle da } \\
\text { espasticidade se mostra bastante eficaz. Contudo, novas } \\
\text { pesquisas acerca deste assunto são necessárias. }\end{array}$ \\
\hline $\begin{array}{l}\text { GOMES, COG; GOLIN, } \\
\text { MO. }\end{array}$ & 2013 & $\begin{array}{l}\text { Descrever o tratamento fisioterapêutico } \\
\text { aplicado em crianças com paralisia } \\
\text { cerebral (PC) tetraparesia espástica } \\
\text { atendidas pelos } \\
\text { acadêmicos do curso de Fisioterapia da } \\
\text { Faculdade de Medicina do ABC - FMABC. }\end{array}$ & $\begin{array}{l}\text { O tratamento aplicado envolveu: padrão de inibição, } \\
\text { manobras para diminuir a hipertonia e facilitação do controle } \\
\text { cervical, de cintura escapular e do rolar. }\end{array}$ \\
\hline
\end{tabular}

Através de estudos bibliográficos, observou-se a importância e os cuidados a serem tomados nos períodos pré-natal, perinatal e pós-natal, pois de acordo com Embiruçu et al. (2015), é através de um pré-natal bem feito e de uma adequada assistência perinatal que os riscos de PC são diminuídos. Ferreira et al. (2016), também traz a visão de que as condições de assistência são precárias nos períodos pré, peri e pós-natal quando se trata de uma população de baixa renda, levando a desigualdade social e tornando mais difícil a vida de uma população que mais precisa de atenção e cuidado. Portanto, a prevenção é a principal medida a ser tomada pois, evita eventos indesejados e consequentemente reduz os casos de paralisia cerebral.

Após o quadro de PC instalado, conforme estudos baseados em Lima et al. (2017), é importante que a estimulação precoce inicie imediatamente após seu diagnóstico, afim de estimular a plasticidade neuronal. $\mathrm{O}$ tratamento para ser eficaz deve ser feito por uma equipe multidisciplinar e o método de tratamento depende do tipo de encefalopatia, porém deve-se escolher o método que promove efeitos duradouros. Ávila et al. (2015), em concordância com Lima et al. (2017), cita que o tratamento fisioterapêutico é paliativo, e é importante ser iniciado precocemente com o intuito de trabalhar a plasticidade neuromotora, aumentando a capacidade funcional através da estimulação de movimentos.

Ao observar os estudos baseados em Gomes et al. (2013), verificou-se que o principal método utilizado é o método Bobath, seguido do Kabat, pois promovem exercícios e estímulos duradouros, necessários para o desenvolvimento das habilidades das funções em relação a neuroplasticidade, diminuindo assim, problemas motores, psicossociais e neuromusculares.

A intervenção precoce deve ser iniciada o mais imediato possível, trabalhando o paciente no todo, principalmente antes dos 3 anos de idade pois nesse período há uma maior plasticidade 
cerebral, possibilitando maiores ganhos no desenvolvimento motor e evitando, prevenindo/minimizando a instalação de padrões posturais e movimentos anormais. Os exercícios executados na intervenção devem ter como finalidade desenvolver a criança na fase em que está, e promover maior independência nas atividades de vida diária (HALLAL et al., 2008; WILLRICH et al., 2008).

Ávila et al. (2014) também cita que o Método Bobath utiliza-se de padrões que influenciam o tônus muscular através de pontos chave de controle que trabalham o controle postural e as atividades funcionais, incentivando e aumentando a capacidade do paciente para mover-se e viver com mais independência.

Há estudos que mostram outros tipos de tratamento fisioterapêutico eficazes como a hidroterapia, que de acordo com Rezende et al. (2015), utiliza-se de efeitos físicos, fisiológicos e cinesiológicos, através de técnicas executadas na piscina aquecida em torno de 32 a $33^{\circ} \mathrm{C}$ com movimentos que são facilitados pela atuação da gravidade diminuída pela força do empuxo. Em PCs, as técnicas mais utilizadas são métodos de Halliwick, Watsu, Bad Ragaz, pois promovem melhora na amplitude de movimento, recrutamento muscular, resistência, treino de deambulação e equilíbrio. A água aquecida promove diminuição de tônus muscular temporariamente e facilita a realização dos movimentos desejados. O principal objetivo da hidroterapia na paralisia cerebral é promover maior independência para o paciente tanto em solo quanto no meio líquido.

Outro método que também contribui para o tratamento do paciente é a equoterapia que conforme Brehn et al. (2015), é um método terapêutico através do auxílio do cavalo que tem como objetivo melhorar as funções motoras, promovendo ganhos físicos, sociais e psicológicos, pois através da mesma são obtidos estímulos que repetem o ritmo, a amplitude e a velocidade através da estimulação vestibular lenta, minimizando assim, o tônus muscular.

Oliveira et al. (2013) cita que através da atuação em cada região do corpo, a cinesioterapia também é importante para o tratamento da PC que deve ser tratada primeiramente de maneira conservadora iniciando o mais precocemente. Cada atividade executada possui características cinesiológicas e biomecânicas necessárias para que a estimulação da musculatura em tempo, velocidade e comprometimento correto.

\section{CONCLUSÕES}

A paralisia cerebral também conhecida por encefalopatia crônica infantil não progressiva trata-se de uma agressão ao sistema nervoso central (SNC) e tem como principal alteração o comprometimento motor, alterações biomecânicas corporais podendo apresentar distúrbios sensitivos, cognitivos, visuais e auditivos. 
Sua classificação depende do tipo e da localização da alteração motora. Podendo ser: espástica, discinética, atáxica, hipotônica e mista.

O presente estudo mostra que a prevenção da PC deve ser principalmente através de um pré-natal rigoroso, afim de, evitar o contágio de doenças e causas que afetarão o feto levando a instalação da patologia, assim como uma boa assistência nos períodos perinatal e pós-natal. Assim como após a instalação da patologia, iniciar a estimulação o mais precocemente para se trabalhar a plasticidade neuronal evitando a instalação de padrões posturais e movimentos anormais.

O fisioterapeuta assim como uma equipe multidisciplinar, cuidadores e pais tem grande importância no tratamento de pacientes com paralisia cerebral, pois através das técnicas aplicadas e ensinadas aos cuidadores e pais, melhora-se o desempenho nas habilidades funcionais, promovendo maior independência nas atividades da vida diária.

\section{REFERÊNCIAS}

ÁVILA, A. S. C; ROCHA, C. Q.. Atuação fisioterapêutica em pacientes com $\mathrm{PC}$ com tetraparesia espástica assimétrica: um estudo de caso. Revista Científica da FAMINAS, Muriaé, v.10, n.2, p.21-27, 2014.

ÁVILA, J. E; RODRIGUES, N. S; SANTOS, R. M; OLIVEIRA A. K. A; SANTOS A. N. L, SAVIAN N. U.. Incidência e mortalidade de paralisia cerebral no Município São Paulo (SP). Revista da Faculdade de Ciências Sociais e Agrárias de Itapeva, Itapeva, v.1, n.1, p.1-7, 2015.

BREHN, M; ALMEIDA, G. M. F.. Benefícios da equoterapia na paralisia cerebral: uma revisão da literatura brasileira. FIEP BULLETIN, Lages, v.85, n.1, p.1-7, 2015.

EMBIRUÇU, E. K.; MONTEIRO, C. B. M.; SILVA, T. D.; REIS, A. O. A.; VALETI, V. E.; OLIVEIRA, A. G.; ABREU, L.C. Paralisia cerebral: revisão de literatura e discussão teórica. In: MONTEIRO, C. B. M.. Paralisia Cerebral. São Paulo: Plêiade, 2015. p.31-56.

FEITOZA, F. B; PEREZ, F. S. B.. Efeitos da intervenção fisioterapêutica na reabilitação de pacientes com paralisia cerebral discinética distônica. Revista da Faculdade Alfredo Nasser, Goiânia, v.1, n.1, p.1-7, 2016.

FERREIRA, L. S. M.; SANTOS, V. B.; PESSOA, P. B.; SILVA, P. S.; SOUSA, S. M. B.; SILVA, L. M.. Análise dos fatores de risco para desenvolvimento de Encefalopatia Crônica não progressiva. Revista Brasileira de Ciências da Saúde, Teresina, v.19, n.2, p.129-134, 2015.

FERREIRA, M. T. S.. Incidência de crianças com paralisia cerebral atendidas na clínica escola de fisioterapia da Unicatólica. Revista Expressão Católica, Quixadá, v.1, n.1, p.87-90, 2016.

GOMES, C. O; GOLIN, M. O.. Tratamento Fisioterapêutico na paralisia cerebral, tetraparesia Espástica, segundo conceito Bobath. Revista Neurocienciências, Santo André, v.21, n.2, p.278-285, 2013.

GUIMARÃES, C. L.; PIZZOLATTO, T. C. O.; COELHO, A. C. S.; FREITAS, S. T. T.. Aspectos clínicos epidemiológicos de crianças com paralisia cerebral assistidas pela clínica escola de Fisioterapia UNIP - São José dos Campos. Journal of the Health Science Institute, São José dos Campos, v.32, n.3, p.281-285, 2014.

HALLAL, C. Z.; MARQUES, N. R.; BRACCIALLI, L. M. P.. Aquisição de habilidades funcionais na área de mobilidade em crianças atendidas em um programa de estimulação precoce. Revista Brasileira de Crescimento e Desenvolvimento Humano, São Paulo, v.18, n.1, p.27-34, 2008.

LIMA, J. F. S. F. A.; MEJIA, D. P. M.. Benefícios da estimulação sensório-motora precoce em crianças com paralisia cerebral (PC). Revista da Faculdade Sul-Americana, Goiânia, v.1, n.1, p.1-12, 2017.

MADEIRA, E. A. A.; CARVALHO, S. G.. Paralisia cerebral e fatores de risco ao desenvolvimento motor: uma revisão teórica. Cadernos de Pós-Graduação em Distúrbios do Desenvolvimento, São Paulo, v.9, n.1, p.142-163, 2009.

MEDEIROS, P. A. B; GRIBOSKI, R. A.. Paralisia cerebral, gestação e parto: uma revisão integrativa. Revista da UnB, Brasília, v.1, n.1, p.1-20, 2015.

MONTEIRO, C. B. M.; SILVA, T. D.; ABREU, L. C.; MASSA, M.; LEÃO, E. K. E. A.. Paralisia Cerebral breve conceituação. In: MONTEIRO, C. B. M.. Realidade virtual na paralisia cerebral. São Paulo: Plêiade, 2011. p.26-43.

OLIVEIRA, L. B.; DANTAS, A. C. L.; PAIVA, J. C.; LEITE, L. P.; FERREIRA, P. H. L.; ABREU, T. M. A.. Recursos fisioterapêuticos na paralisia cerebral pediátrica. Catussaba, Potiguar, v.2, n.2, p.25-37, 2013.

RESENDE, A. S.; CRUZ, P. F.. Efeitos da Hidroterapia em crianças com paralisia cerebral: uma revisão. Pontifícia Universidade Católica de Goiás, Goiânia, v.1, n.1, p.1-12, 2015.

SANTOS, A. F. Paralisia cerebral: uma revisão da literatura. Revista Unimontes Científica, Montes Claros, v.16, n.2, p.6782, 2014 
SILVA, R.; CAON, G.; RIBEIRO, J.; VARGAS, C. R.. Função motora grossa e fatores etiológicos associados à paralisia cerebral. Fitness \& Perfomance Journal, Rio de Janeiro, v.8, n.5, p.372-377, 2009.

TELES, M. S.; MELLO, E. M. C. L.. Toxina botulínica e fisioterapia em crianças com paralisia cerebral Espástica: revisão bibliográfica. Fisioterapia em Movimento, Curitiba, v.24, n.1, p.181-190, 2011.
WILLRICH, A.; AZEVEDO, C. C. F.; FERNANDES, J. O..

Desenvolvimento motor na infância: influência dos fatores de risco e programas de intervenção. Revista Neurociência, Porto Alegre, v.1, n.1, p.1-6, 2008.

ZANINI, G.; CEMIN, N. F.; PERALLES, S. N.. Paralisia cerebral: causas e prevalências. Fisioterapia em Movimento, Curitiba, v.22, n.3, p.375-381, 2009.

A CBPC - Companhia Brasileira de Produção Científica (CNPJ: 11.221.422/0001-03) detém os direitos materiais desta publicação. Os direitos referem-se à publicação do trabalho em qualquer parte do mundo, incluindo os direitos às renovações, expansões e disseminações da contribuição, bem como outros direitos subsidiários. Todos os trabalhos publicados eletronicamente poderão posteriormente ser publicados em coletâneas impressas sob coordenação da Sustenere Publishing, da Companhia Brasileira de Produção Científica e seus parceiros autorizados. Os (as) autores (as) preservam os direitos autorais, mas não têm permissão para a publicação da contribuição em outro meio, impresso ou digital, em português ou em tradução. 